\title{
Analogue Filters and their Applications in Control Systems
}

\author{
Karush Suri \\ Department of Electronics and Communication Engineering, Amity University, Uttar Pradesh
}

\begin{abstract}
Control systems find their applications in various complex consortiums of the modern technical era. From navigation and mapping to voice monitoring these networks play a significant role in the field of electronic devices. However, the design of these systems is quite complex and is made use of with the help of a number of filtering units. Mainly the analogue filters, they provide the sufficient amount of data filtering by using a number of well-defined algorithms. In principle, the design of such filters can be achieved entirely in terms of the electronic counterparts of mechanical quantities, with kinetic energy, potential energy and heat energy corresponding to the energy in inductors, capacitors and resistors respectively. Modern techniques also include the use of various modified filters essentially used for special purposes. These are fabricated in order to target a specific function and filter only a specific kind of data. One such filter being used these days is the advanced Kalman filter. The filter is altered in order to carry out single-handed operations and block a number of unrequired signals in the arena of telecommunication networking.
\end{abstract}

Keywords: Gaussian Control Problem, Linear Dynamic System, Linear Quadratic Estimation, Proportional-Integral Derivative

\section{Introduction}

Analogue filters are a basic building block of signal processing much used in electronics. Amongst their many applications are the separation of an audio signal before application to bass, mid-range and tweeter loudspeakers; the combining and later separation of multiple telephone conversations onto a single channel; the selection of a chosen radio station in a radio receiver and rejection of others. Analogue filters have played an important part in the development of electronics. Especially in the field of telecommunications, filters have been of crucial importance in a number of technological breakthroughs and have been the source of enormous profits for telecommunications companies. It should come as no surprise, therefore, that the early development of filters was intimately connected with transmission lines. Transmission line theory gave rise to filter theory, which initially took a very similar form, and the main application of filters was for use on telecommunication transmission lines. However, the arrival of network synthesis techniques greatly enhanced the degree of control of the designer.It is possible to design linear analogue mechanical filters using mechanical components which filter mechanical vibrations or acoustic waves. While there are few applications for such devices in mechanics per se, they can be used in electronics with the addition of transducers to convert to and from the electrical domain. Indeed, some of the earliest ideas for filters were acoustic resonators because the electronics technology was poorly understood at the time.

\section{1 Filter Design}

Despite the rise of digital filters, analogue domain filters are still an essential part of many electronic systems; for example in high frequency circuits where high frequency and high resolution ADCs are costly. Increasingly analogue filters are also being used in low frequency applications such as in analogue signal processing units in wearable physiological sensors. For such battery powered physiological monitors power efficiency is essential, and the use of analogue signal processing allows the low dynamic ranges and signal-to-noise ratios of many biological signals to be exploited. It is well known that analogue filters are potentially much more power efficient than their digital counterparts in such circumstances.At the circuit level, many different analogue filter topologies are available, with cascades of second order sections and orthonormal ladder filters $^{[1]}$ being popular choices for on-chip implementation. However, at both very high and very low frequencies parasitic components become significant in the circuit design of the filter. Also, for low power and low voltage circuits the use of transistors operating in the weak inversion region is unavoidable (due to the intrinsically low drain currents provided), although this comes at the cost of decreased transistor current matching. For robust analogue filters in these situations it is essential that the sensitivity of the filter transfer function to non-exact component values is minimised: device mismatch and parasitic components should not change the shape of the implemented frequency response. Filters based upon LC ladder prototypes, where the filter transfer function is implemented by lossless inductor and capacitor elements connected between terminating resistances, are known to have very low sensitivities to non-exact component values. Indeed doubly terminated LC ladders with equal terminating resistances are "very nearly an optimum realization for filter functions".

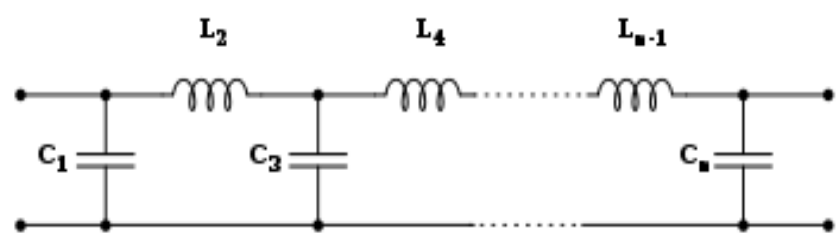

\subsection{Control Loop Pre-Filtering}

In designing feedback control systems, it's often necessary to use a compensator to meet stability margins and set fundamental speed and damping. The compensator will often contain lead elements which introduce zeros into the closed loop transfer function. The presence of zeros in either 


\section{International Journal of Science and Research (IJSR) \\ ISSN (Online): 2319-7064 \\ Index Copernicus Value (2013): 6.14 | Impact Factor (2014): 5.611}

the system or compensator may cause overshoot in the transient response for the closed loop system, the size depending upon the relative position of the zeros and closed loop poles within the complex plane.

The PI (proportional-integral) ${ }^{[2]}$ and PID (proportionalintegral derivative) compensators are common examples of compensators that introduce zeros and which may lead to overshoot. But even with the introduction of zeros, it's possible to reduce or eliminate overshoot in the closed loop system from input commands by applying a pre-filter to the loop input command outside of the feedback loop. The procedure is simple:

- Determine the zeros of the closed loop system

- Design a lowpass filter in which the poles match the zeros of the closed loop system

- Apply the lowpass filter to the input command outside the closed loop system

The poles of the pre-filter essentially cancel the zeros of the closed loop system. Another way to look at it is that the prefilter prevents the admission of frequencies into the input command that the closed loop system is unable to cope with in terms of reaching a target value without overshoot. It is important to note that the pre-filter does not change stability of the overall system considering the pre-filter itself is stable, and the closed loop is linear and stable. If designed properly, the pre-filter can effectively eliminate overshoot from input commands. Even so, a pre-filter will not solve overshoot considering the following circumstances:

- The zeros of the closed loop system are not stationary with respect to other system parameters or with time. This can occur in non-linear or time varying systems

- System input commands can be pre-filtered, however system disturbances usually cannot be pre-filtered. Thus the pre-filter is practical for tracking controls or for preventing overshoot while changing the set-point for a regulator. Pre-filters in general will not prevent overshoot due to disturbance.

Finally, If the overshoot of the closed loop system is relatively large, designing a pre-filter by the previously outlined technique will likely result in an overall system response that is accordingly slow. In this case, it is better to first change the closed loop dynamics by adjusting gains or choosing a different compensator structure before considering application of a pre-filter.

\subsection{Kalman Filtering}

Kalman filtering, also known as linear quadratic estimation $(\mathrm{LQE})^{[3]}$, is an algorithm that uses a series of measurements observed over time, containing statistical noise and other inaccuracies, and produces estimates of unknown variables that tend to be more precise than those based on a single measurement alone. The filter is named after Rudolf E. Kalman, one of the primary developers of its theory. It has numerous applications in technology. A common application is for guidance, navigation and control of vehicles, particularly aircraft and spacecraft. Furthermore, the Kalman filter is a widely applied concept in time series analysis used in fields such as signal processing and econometrics. Kalman filters also are one of the main topics in the field of robotic motion planning and control, and they are sometimes included in trajectory optimization. The Kalman filter has also found use in modeling the central nervous system's control of movement. Due to the time delay between issuing motor commands and receiving sensory feedback, use of the Kalman filter provides the needed model for making estimates of the current state of the motor system and issuing updated commands.

The algorithm ${ }^{[4]}$ works in a two-step process. In the prediction step, the Kalman filter produces estimates of the current state variables, along with their uncertainties. Once the outcome of the next measurement (necessarily corrupted with some amount of error, including random noise) is observed, these estimates are updated using a weighted average, with more weight being given to estimates with higher certainty. The algorithm is recursive. It can run in real time, using only the present input measurements and the previously calculated state and its uncertainty matrix; no additional past information is required.It does not require any assumption that the errors are Gaussian. However, the filter yields the exact conditional probability estimate in the special case that all errors are Gaussian-distributed.

The Kalman filter is an efficient recursive filter that estimates the internal state of a linear dynamic system from a series of noisy measurements. It is used in a wide range of engineering and econometric applications from radar and computer vision to estimation of structural macroeconomic models, and is an important topic in control theory and control systems engineering. Together with the linear quadratic regulator $(\mathrm{LQR})^{[5]}$, the Kalman filter solves the linear quadratic Gaussian control problem (LQG). The Kalman filter, the linear-quadratic regulator and the linearquadratic-Gaussian controller are solutions to what arguably are the most fundamental problems in control theory.In most applications, the internal state is much larger (more degrees of freedom) than the few "observable" parameters which are measured. However, by combining a series of measurements, the Kalman filter can estimate the entire internal state.In Dempster- Shafter theory, each state equation or observation is considered a special case of a linear belief function and the Kalman filter is a special case of combining linear belief functions on a join-tree or Markov tree. Additional approaches include belief filters which use Bayes or evidential updates to the state equations. 


\section{Block Diagram representation of Kalman Filter}

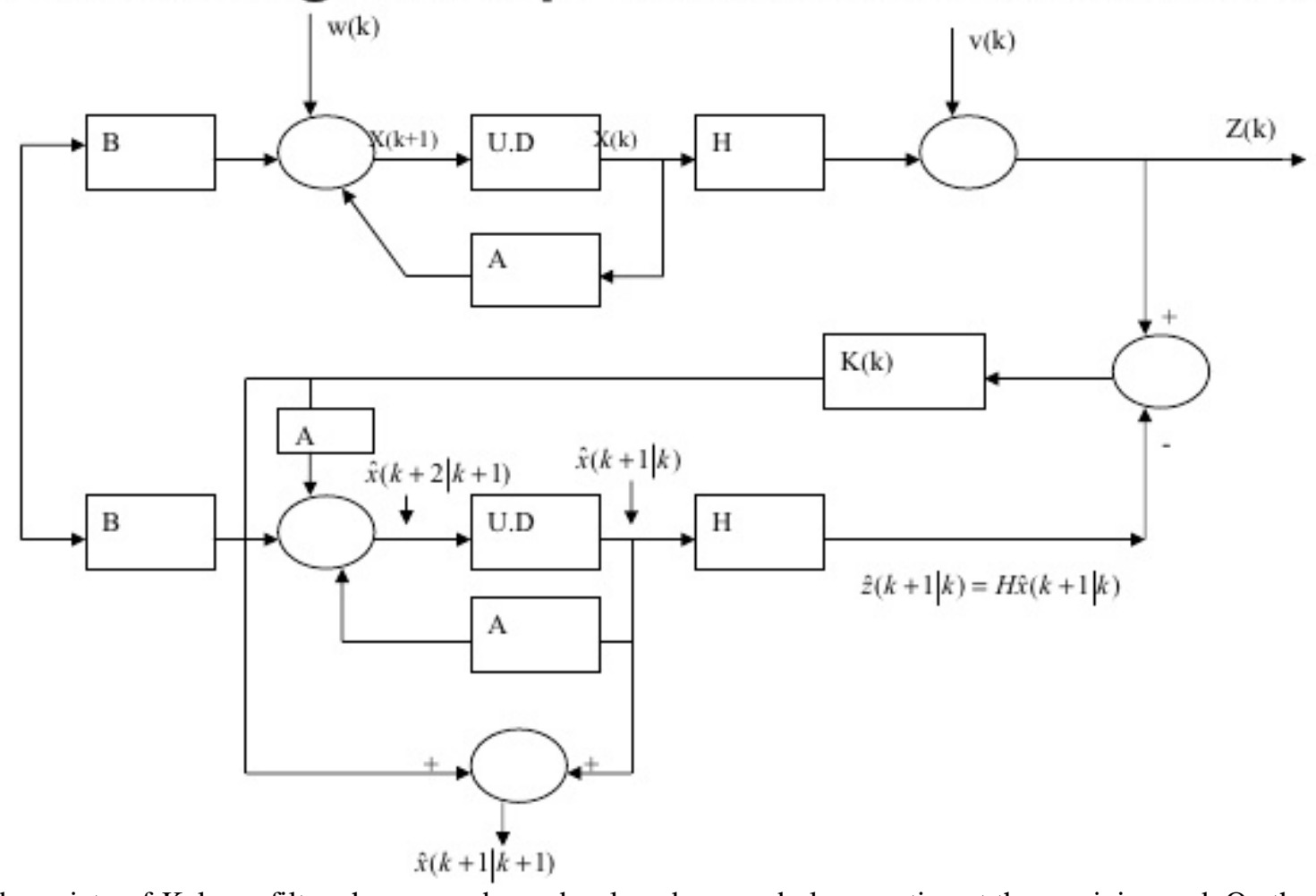

A wide variety of Kalman filters have now been developed, from Kalman's original formulation, now called the "simple" Kalman filter, the Kalman- Bucy filter, Schmidt's "extended" filter, the information filter, and a variety of "square-root" filters that were developed by Bierman, Thornton and many others. Perhaps the most commonly used type of very simple Kalman filter is the phase locked loop, which is now ubiquitous in radios, especially frequency modulation (FM) radios, television sets, satellite communication receivers, outer space communications systems, and nearly any other electronics communications equipment.

This filtering technique helps in the processing of a wellaccepted control system. The design being complex is realised and then applied to various application scenarios. Proper testing of the filtering unit is required before its application. The filter is then used for removing noise, devising complex network systems, image filtering and cancelling unrequired data. Such a technique is implied with great care and awareness. A proper analysis of the situation and the data to be eliminated is carried out and after this inspection the filter is put to use. Quick filtering, effective output and production of a durable control network are the main advantages of this filtering unit.

\section{Conclusion}

Control filter systems are of great significance in the modern era of telecommunication. Without proper fabrication of an effective analogue filtering unit it is difficult to encrypt data signals and process them at higher rates. Another important characteristic feature of these filters is that their functioning in complex control systems is unique and these possess the ability to seperate unrequired data from processing signals at effective rates. This helps in efficient transmission as well as orderly reception at the receiving end. On the other hand, the wide variety of situation specified filters such as the Kalman filters is also an emerging trend in the networking field. Different specified singly-functioning units can be substituted in place of a bank of primitive filtering components. This will help in higher transmission rates and better realising of the network at hand.

\section{References}

[1] Orthonormal Ladder Filters', D.A. Johns, W.M. Snelgrove, A.S. Sedra, IEEE Transactions on Circuits and Systems, March 1989

[2] Williams, Arthur B \& Taylor, Fred J (1995). Electronic Filter Design Handbook.

[3] 'Robust Extended Kalman Filtering in Hybrid Positioning Applications', Tommi Perala and Robert Piche, IEEE, Positioning, Navigation and Communication, 2007.

[4] Kalman, R. E. (1960). "A New Approach to Linear Filtering and Prediction Problems". Journal of Basic Engineering 82: 35

[5] Roweis, S; Ghahramani, Z (1999). "A unifying review of linear gaussian models". Neural computation 11 (2): 305-45.

\section{Author Profile}

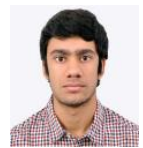

Born on $17^{\text {th }}$ March, 1997, Karush Suri is currently resides in Noida with his famliy. He completed his schooling from Amity International School, Noida and is currently pursuing his Bachelors in Technology in Electronics and Communication Engineering from Amity University, Uttar Pradesh. Karush has been an active participant in the field of research and has a keen interest in the electronic functionings. His work has been published in Internal Journal of Electronics and Communication Engineering ( IJECE) also. 